\title{
A Study of PCAC for the Nonperturbative Improvement of the Wilson Action
}

\author{
Aida X. El-Khadra \\ Department of Physics, University of Illinois, 1110 W. Green St., Urbana, IL 61801-3080
}

\begin{abstract}
We present an exploratory study for the nonperturbative determination of the coefficient of the $\mathcal{O}(a)$ improvement term to the Wilson action, $c_{\mathrm{SW}}$. Following the work by Lüscher et al., we impose the PCAC relation as a nonperturbative improvement condition on $c_{S W}$, without, however, using the Schrödinger functional in our calculation.
\end{abstract}

\section{INTRODUCTION}

Much progress has been made in recent years in lattice QCD with the help of improved actions. With better numerical tests, we also need better determinations of the coefficients of the improvement terms. We concentrate here on $\mathcal{O}(a)$ improvement for the fermion action, and start with the Sheikholeslami-Wohlert action [1],

$$
\begin{aligned}
S^{\mathrm{lat}} & =a^{4} \sum_{x}\left[m_{0} \bar{\psi} \psi+\bar{\psi} \not D^{L} \psi-\frac{1}{2} \operatorname{ar} \bar{\psi} \Delta^{L} \psi\right. \\
& \left.+\frac{i}{4} c_{S W} \bar{\psi} \sigma_{\mu \nu} F_{\mu \nu}^{L} \psi\right]
\end{aligned}
$$

where $D_{\mu}^{L}$ and $\Delta^{L}$ are discretizations of the covariant derivative and the laplacian, and $F_{\mu \nu}^{L}$ is the cloverleaf approximation to the $F_{\mu \nu}$ operator. At tree-level, $c_{S W}=1$ [1]. With tadpole improvement,

$c_{S W}=\frac{1}{u_{0}^{3}}$.

The one-loop correction is also known [2]:

$c_{S W}=\frac{1}{u_{0}^{3}}\left[1+0.20 \alpha_{V}+\mathcal{O}\left(\alpha_{V}^{2}\right)\right]$,

where $u_{0}$ is defined from the plaquette, $u_{0}=$ $\left\langle\operatorname{tr} U_{\square}\right\rangle$. Finally, a recent nonperturbative determination [3] parametrizes the improvement coefficient as:

$c_{S W}=\frac{1-0.656 g_{0}^{2}-0.152 g_{0}^{4}-0.054 g_{0}^{6}}{1-0.922 g_{0}^{2}}$,

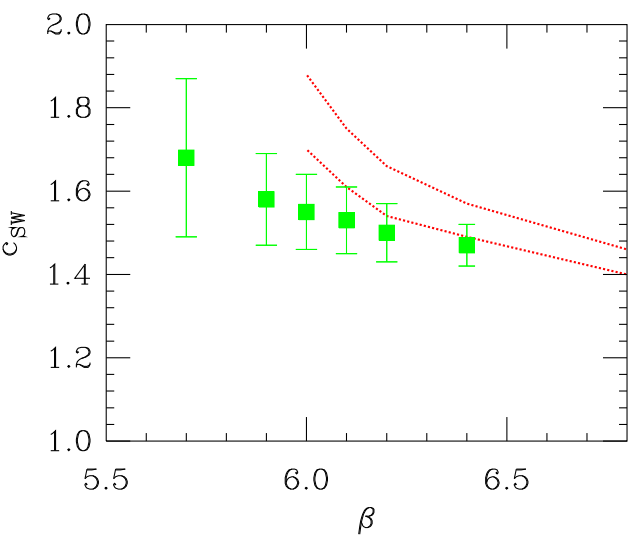

Figure 1. $c_{S W}$ in comparison. $\square$ : one-loop result of Eq. (3), with $q^{*} \simeq 1 / a$ and $\alpha_{V}$ as determined from the plaquette [4]; the error bars correspond to $\pm \alpha_{V}^{2}$. - : nonperturbative $c_{S W}$ from Ref. [3]; the error band is taken as $\pm 2 \%$ at $\beta=6.8$ and $\pm 5 \%$ at $\beta=6.0$.

for $\beta \equiv 6 / g_{0}^{2} \geq 6.0$. This result has an unknown $\mathcal{O}(a)$ error, which contributes an error of $\mathcal{O}\left(a^{2}\right)$ to the action. It is therefore interesting to find alternative nonperturbative determinations of $c_{S W}$ to explore this uncertainty further.

Fig. 1 shows a comparison between the oneloop and the nonperturbative results for $c_{S W}$ as a function of $\beta$. The comparison shows that the perturbative and nonperturbative results for $c_{S W}$ are in reasonable agreement, once the uncertainties are taken into account. 


\section{THE PCAC RELATION}

The continuum PCAC relation, $\partial_{\mu} A_{\mu}(x)=$ $2 m P(x)$, is modified on the lattice, due to lattice spacing artifacts. With $\mathcal{O}(a)$ improvement, the axial current is

$A_{\mu}^{I}(x)=A_{\mu}(x)+c_{A} \partial_{\mu} P(x)+\mathcal{O}\left(a^{2}\right)$

where the improvement coefficient $c_{A}$ also needs to be determined. The PCAC relation then is

$\partial_{\mu}\left\langle A_{\mu}^{I}(x) \Pi(0)\right\rangle=2 m\langle P(x) \Pi(0)\rangle+\mathcal{O}\left(a^{2}\right)$,

This equation is valid for any (pion) operator, and can be used to find conditions that determine $c_{S W}$ and $c_{A}$, for example, by varying the $\Pi$ operators, or the euclidean time dependence, or the boundary conditions.

The Schrödinger functional in Ref. [3] provides a nice condition for $c_{S W}$. In this case, the boundary conditions induce a color background field, so that the vertex, $\bar{\psi} \sigma_{\mu \nu} F_{\mu \nu} \psi$, is sensitive to the boundary conditions. We want to explore Eq. (6) without using the Schrödinger functional in our numerical calculation, to make use of existing lattices and shed light on the $\mathcal{O}(a)$ error associated with nonperturbative determinations of $c_{S W}$.

An alternative to the Schrödinger functional boundary conditions is the momentum dependence of the two-point functions in Eq. (6). This can be seen as follows. The operator $\psi D^{2} \psi$ is redundant at order $\mathcal{O}(a)$ [1], and can be written as

$\bar{\psi} \not D^{2} \psi=\bar{\psi} D^{2} \psi-\frac{i}{2} \bar{\psi} \sigma_{\mu \nu} F_{\mu \nu} \psi$

In the lattice action the first term is the Wilson term (a discretized laplacian) and the omission of the second term from the Wilson action gives rise to the well known $\mathcal{O}(a)$ errors. In the Sheikholeslami-Wohlert action the second term is added to the lattice action, however, the $F_{\mu \nu}$ operator is discretized using plaquettes arranged in a "clover-leaf". Thus, the second term acquires a coefficient, $c_{S W}$, which is a function of $\alpha_{s}$ (and $m_{0}$ ). Going to Fourier space, the momentum dependence is then sensitive to $c_{S W}$. Thus, we can use operators with different momenta, $\Pi(\vec{p})$ (or by translational invariance, $\partial_{\mu} A_{\mu}^{I}(\vec{p})$ ), in Eq. (6) to determine $c_{S W}$.
Following Ref. [3], we write Eq. (6) as

$m(t)=\frac{1}{2} \frac{\partial f_{A_{\mu}}(t)+c_{A} \partial^{2} f_{P}(t)}{f_{P}(t)}=r(t)+c_{A} s(t)(8)$

with

$f_{A_{\mu}}(t)=\left\langle A_{\mu}(t) \Pi(0)\right\rangle, f_{P}(t)=\langle P(t) \Pi(0)\rangle$

Defining $m^{\prime}(t)$ using a different operator $\Pi^{\prime}$, it follows from Eq. (6) that $m-m^{\prime}=\mathcal{O}\left(a^{2}\right)$, if $c_{S W}$ and $c_{A}$ are tuned to their correct values. In the combination

$M\left(t, t^{\prime}\right)=r(t)-s(t) \frac{r\left(t^{\prime}\right)-r^{\prime}\left(t^{\prime}\right)}{s\left(t^{\prime}\right)-s^{\prime}\left(t^{\prime}\right)}$

$c_{A}$ drops out. Furthermore [3], $M=m+\mathcal{O}\left(a^{2}\right)$, and $\Delta M\left(t, t^{\prime}\right)=M\left(t, t^{\prime}\right)-M^{\prime}\left(t, t^{\prime}\right)=\mathcal{O}\left(a^{2}\right)$, if $c_{S W}$ is tuned to its correct nonperturbative value.

\section{RESULTS}

The lattices used in this calculation were originally generated for a different purpose. They were chosen for this exploratory study for calculational convenience. In particular, we have lattices with only two values of the improvement coefficient, $c_{S W}=0$ (Wilson case) and $c_{S W} \approx 1 / u_{0}^{3}$. At $\beta=5.5\left(8^{3} \times 16\right)$ we have 500 (quenched) configurations with one quark mass per $c_{S W}$ value. At $\beta=5.9\left(16^{3} \times 32\right)$ we have 350 (quenched) configurations at $c_{S W}=1.50$ only, but for a range of quark masses.

Fig. 2 shows a comparison of the time dependence of $\Delta M$ for two different values of $c_{S W}$ at $\beta=5.5$. It appears that $\Delta M$ is different for the two values of $c_{S W}$ and that $\Delta M$ is closer to zero for $c_{S W}=1.69$. On both lattices, the quark mass roughly corresponds to the strange quark mass, $\kappa \approx \kappa_{s}$. This large quark mass was chosen, because at smaller quark masses the $\beta=5.5$ lattices suffer from an increasing number of exceptional configurations [5].

We study the quark mass dependence of $\Delta M$ at $\beta=5.9$, as shown in Fig. 3. We find that the mass dependence is smaller than our statistical errors, consistent with previous results [3, 6]. The errors increase with decreasing quark mass. Finally, Fig. 目 shows our result for $\Delta M$ versus $c_{S W}$ at $\beta=5.5$. 


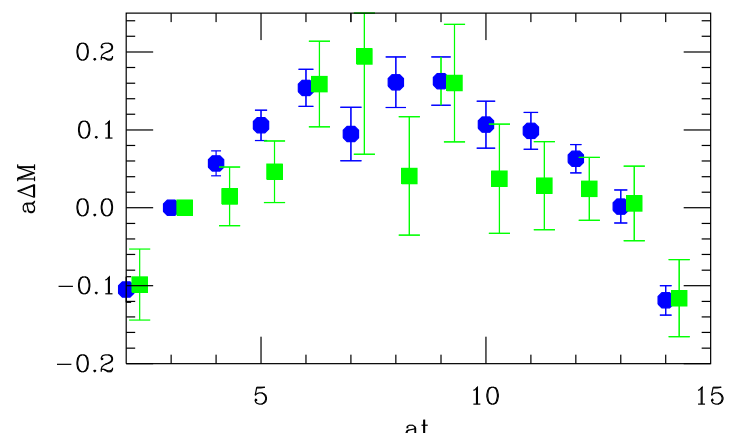

Figure 2. $\Delta M\left(t, t^{\prime}=3\right)$ vs. $t$ at $\beta=5.5$. blue $\circ$ : $c_{S W}=0$ and $\kappa=0.169$, green $\square: c_{S W}=1.69$ and $\kappa=0.1423$. The squares are offset for readability.

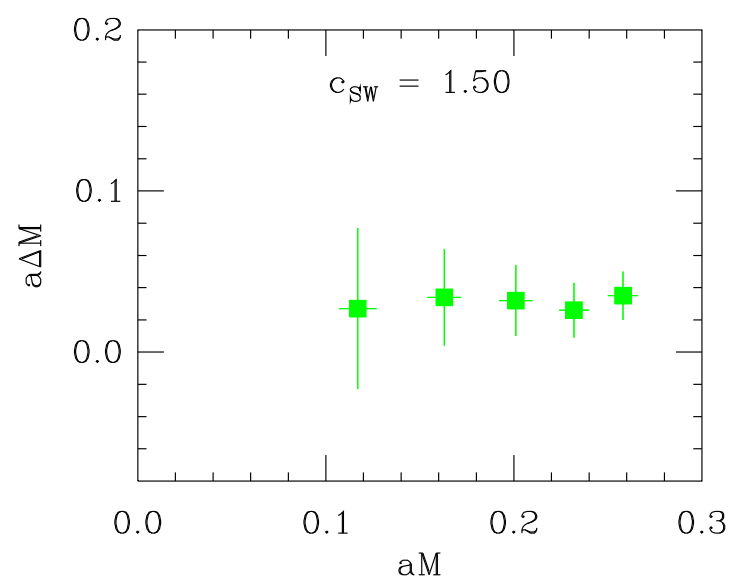

Figure 3. $\Delta M$ vs. $a M$ at $\beta=5.9$, $c_{S W}=1.50$, and (from right to left) $\kappa=$ $0.1382,0.1385,0.1388,0.1391,0.1394$.

In conclusion, we showed that the momentum dependence appears to be a sensitive tool to determine $c_{S W}$ nonperturbatively. $\Delta M$ is independent of the light-quark mass, albeit within large statistical errors. Our results indicate that the nonperturbative value for $c_{S W}$ is larger than that suggested by tadpole improvement (with the plaquette), $c_{S W}>1 / u_{0}^{3}$. This is, of course, consistent with the nonperturbative determinations of $c_{S W}$ based on the Schrödinger functional. Clearly, more work must be done in order to use our method for a quantitative, nonperturbative determination of $c_{S W}$.

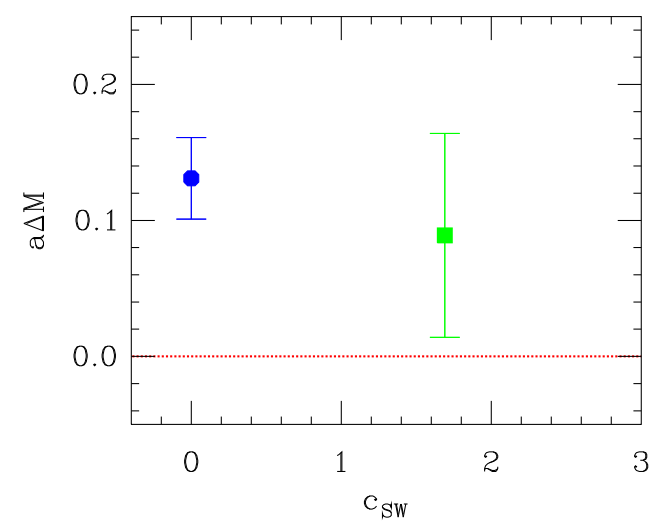

Figure 4. $\Delta M$ vs. $c_{S W}$ at $\beta=5.5$. At $c_{S W}=0$ $\kappa=0.169$ and at $c_{S W}=1.69 \kappa=0.1423$.

\section{ACKNOWLEDGEMENTS}

I thank P. Lepage and M. Lüscher for discussions, and of course my collaborators, Andreas Kronfeld, Paul Mackenzie, Sinéad Ryan, and Jim Simone. The numerical work presented here was performed on the Fermilab ACPMAPS computer. This work is supported in part through the DOE OJI program under grant no. DE-FG0291ER40677 and by a fellowship from the Alfred P. Sloan foundation. Finally, I thank the organizers for an enjoyable conference and especially Ken Bowler and Richard Kenway for excellent advice on hillwalking in the Scottish Highlands.

\section{REFERENCES}

1. B. Sheikholeslami and R. Wohlert, Nucl. Phys. B259 (1985) 572.

2. R. Wohlert, Ph.D. thesis, DESY preprint 87069 (1987), unpublished; M. Lüscher and P. Weisz, Nucl. Phys. B479 (1996) 429.

3. M. Lüscher, et al., Nucl. Phys. B491 (1997) 323; Nucl. Phys. B (Proc. Suppl.) 53 (1997) 905, Nucl. Phys. B478 (1996) 365.

4. P. Lepage and P. Mackenzie, Phys. Rev. D48 (1992) 2250.

5. E. Eichten, plenary talk, these proceedings.

6. R. Edwards, these proceedings; T. Klassen, these proceedings; hep-lat/9705025. 